\section{SHAPE AND DIMENSIONAL} ANALYSIS OF LATTICE
STRUCTURES PRODUCED BY
SELECTIVE LASER MELTING
RADEK VRANA', ONDREJ VAVERKA', DANIEL KOUTNYY,
KATERINA DOCEKALOVA ${ }^{2}$, DAVID PALOUSEK ${ }^{1}$

${ }^{1}$ Brno University of Technology, Faculty of Mechanical Engineering, Brno, Czech Republic

${ }^{2}$ Honeywell International s.r.o, Praha, Czech Republic DOI: 10.17973/MMSJ.2020_06_2020013

e-mail : Radek.Vrana@vut.cz

Mechanical properties of lattice structures produced by Selective Laser Melting (SLM) are hardly predictable using a numerical simulation. It is caused by procedural inaccuracies of the SLM, such as shape and dimension deviations which are usually not included in a simulation. In order to quantify their influence, lattice structure specimens were produced by SLM and digitized in the whole volume using $\mu \mathrm{CT}$. The shape and dimensional analyses were performed in each strut of lattice structure using the developed script in GOM Inspect software. Based on statistical analysis, the global dimension parameters of lattice structures were obtained and used in FEA models.

The shape analysis showed large deviations in diameters of inscribed and circumscribed cylinders which implied the elliptic shape of the lattice structure. It makes the lattice structure significantly stiffer mainly under compression loading which was confirmed by FEA comparison of circular and elliptical geometry of the lattice structure.

KEYWORDS

Selective Laser Melting (SLM), lattice structures, shape analysis, dimension analysis, digitization, finite element analysis

\section{INTRODUCTION}

Selective Laser Melting (SLM) is an additive technology which allows production of metallic components - layer by layer directly from 3D CAD data using a high power YLR fibre laser and a metal powder. The process starts by depositing of the metal powder on a platform. Then the powder is melted at inert atmosphere by a laser beam, the platform is lowered by one-layer thickness and the next layer is fabricated directly on the previous one. The process is repeated until the whole part is produced [Schleifenbaum 2010]. The main advantage of the additive production is the possibility to produce a complex geometry such as lattice structures which cannot be achieved conventionally [Leary 2016]. The lattice structures are usually cellular structures composed of regularly repeated struts or complex shape unit cells [Vrana 2016]. Due to great weight to load ratio, they have the potential for application in aerospace or space industry.

In term of SLM production, the lattice structure is specific geometry which is hard to produce using default process parameters designed mainly for volume parts. It can lead to production inaccuracies as porosity, high surface roughness or shape and dimensional deviation. Therefore, the process parameters must be optimized directly for lattice structure production [Vrana 2018a]. Qiu et al. investigated the influence of the laser power (LP) and the scanning speed $(\boldsymbol{L S})$ on the diameter, shape, and porosity of the struts made of AISi10Mg material. The linear dependence between the strut diameter and the increasing $L P$ was found. The results show that according to the used $\boldsymbol{L} \boldsymbol{P}$, the real diameter of strut changed from $260 \mu \mathrm{m}$ to $500 \mu \mathrm{m}$ for the nominal diameter of $300 \mu \mathrm{m}$ [Qiu 2015]. Koutny et al. examined the influence of the strut orientation on the real strut dimensions. The strut specimens were measured by a 3D optical scanner and evaluated by fitting maximum inscribed cylinders inside the struts which represents the largest homogenous diameters not influenced by high surface roughness on the strut downskin. The results show that the surface roughness rapidly increases at low strut inclination $\left(<45^{\circ}\right)$ and at low strut diameters [Koutny 2014]. Leary et al. investigated a manufacturability and surface roughness of the struts with the orientation typical for micro strut-lattice structures. They found that the surface roughness on the strut down-skin surface is significantly higher due to a heat transfer and sticking of the surrounding powder on the strut down-skin. The surface roughness is strongly dependent on the strut orientation [Leary 2016].

The previous studies showed that SLM technology allows production of lattice structure; however, during production, significant inaccuracies occurred. This study aimed primarily on evaluation of a real shape and dimension deviations measured on large number of the struts within lattice structure. The results help to improve accuracy of the FEA models in the future.

\section{MATERIAL AND METHODS}

\subsection{Lattice Structure Specimens}

To determine the procedural deviations of the BCC lattice structures made by SLM, the lattice structure cube specimens with different strut diameters were produced (Figure 1). The dimensional parameters of the cubes were designed to keep constant relative density $5 \%$ for all specimens according to Table 1; where $\boldsymbol{d}$ is diameter of the lattice structure struts; $\boldsymbol{a}$ is the size of the lattice unit cell; $\boldsymbol{N}$ is the number of the unit cells in the rows and columns and $\boldsymbol{I}$ is the size of the whole lattice cube (Figure 2).

\begin{tabular}{|c|c|c|c|c|}
\hline $\begin{array}{c}\text { Param. - } \\
\text { Specimens }\end{array}$ & $\begin{array}{c}\mathbf{d} \\
(\mathrm{mm})\end{array}$ & $\begin{array}{c}\mathrm{a} \\
(\mathrm{mm})\end{array}$ & $\begin{array}{c}\mathbf{N} \\
(-)\end{array}$ & $\begin{array}{c}\text { I } \\
(\mathbf{m m})\end{array}$ \\
\hline LC-01 & 0.6 & 6 & 7 & 42 \\
\hline LC-02 & 0.8 & 8 & 5 & 40 \\
\hline LC-03 & 1 & 10 & 4 & 40 \\
\hline
\end{tabular}

Table 1. The geometrical parameters of the tested lattice structures cubes

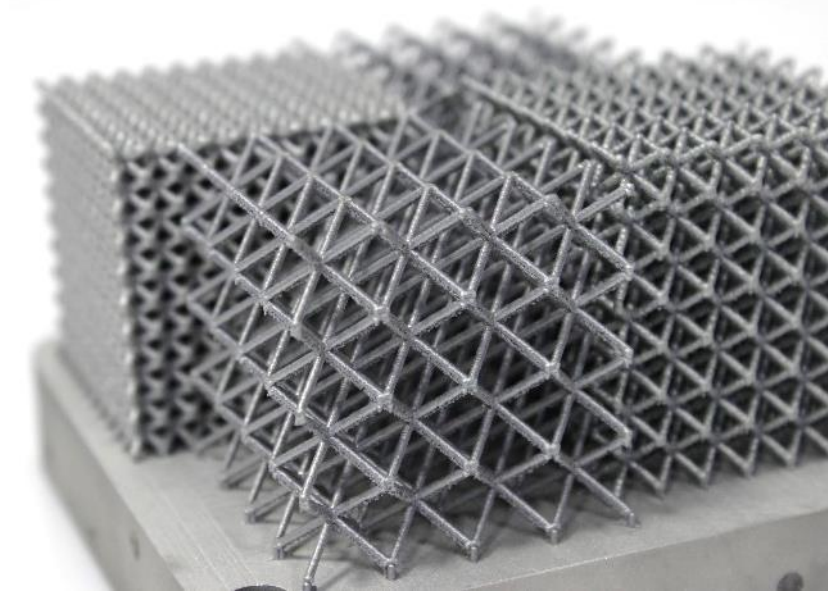

Figure 1. Produced lattice structures on the platform 


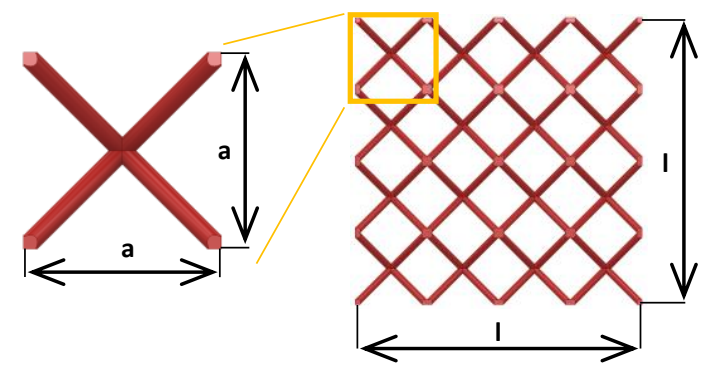

Figure 2. Schema of the BCC lattice structure with geometrical parameters

All samples were manufactured using the SLM machine (SLM $280 \mathrm{HL}, \mathrm{SLM}$ Solution $\mathrm{GmbH}$ ) equipped with $400 \mathrm{~W}$ YLR laser which is focused to the diameter of $82 \mu \mathrm{m}$ and has a Gaussian shape with a continuous wave. Laser scanning speed may reach up to $10,000 \mathrm{~mm}$. $\mathrm{s}^{-1}$ and maximum build envelope is $280 \times 280 \times 350 \mathrm{~mm}$. During the production process, $\mathrm{N}_{2}$ atmosphere was used in the chamber and the oxygen level was kept under $0.2 \%$. Before fabrication, the platform was preheated to $150{ }^{\circ} \mathrm{C}$. The standard SLM process parameters from SLM Solution $\mathrm{GmbH}$ were used for fabrication, specifically:

- for the border strategy: $\mathbf{L S}=600 \mathrm{~mm} . \mathrm{s}^{-1} ; \mathbf{L P}=300 \mathrm{~W}$

- for the volume strategy: $\mathbf{L S}=1150 \mathrm{~mm} \cdot \mathrm{s}^{-1} ; \mathbf{L P}=350 \mathrm{~W}$

\subsection{Shape Analysis Using Semi-automatic Script}

The lattice structures are usually a complex shape which is difficult to measure by conventional way; therefore, the computed tomography was used $(\mu \mathrm{CT}, \mathrm{GE}$ phoenix $v$ (tome $\mid x$ L240). The main parameters of the X-ray tube were voltage of $130 \mathrm{kV}$, current of $100 \mu \mathrm{A}$, and a filter of $0.5 \mathrm{~mm}$ thin copper plate. The measured data were obtained with linear voxel size accuracy $I / 1000$ and were reconstructed using the back-projection algorithm in the Datos reconstruction software. All subsequent post-processing was performed in the software VGStudio MAX 3.1.

The digitized surface data of the lattice structures were used together with CAD data for evaluation of real geometry using the semi-automatic script (Figure 3) which works in the following steps (Figure $4 a-4 d$ ):

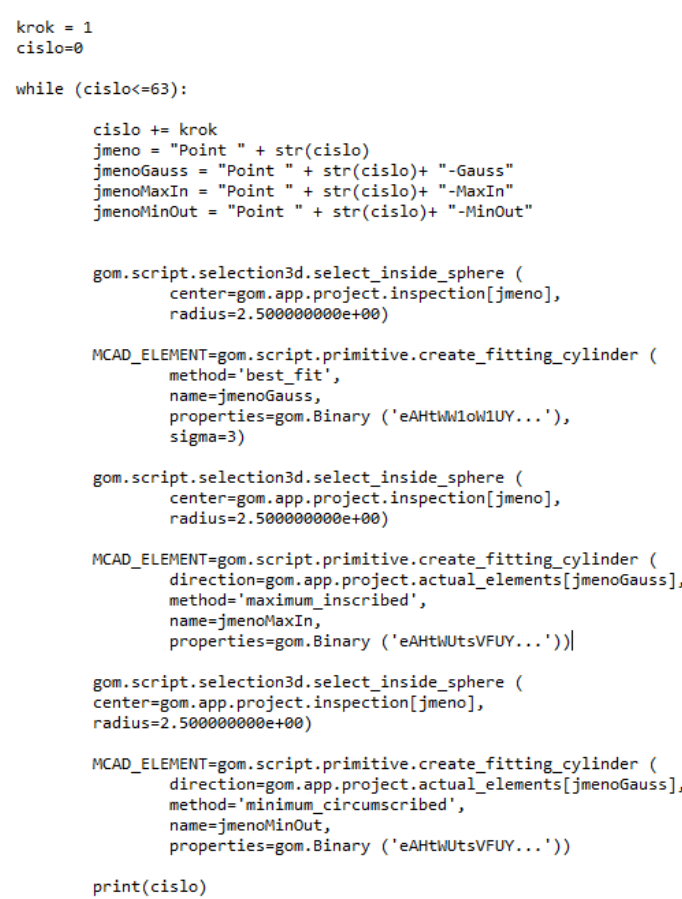

Figure 3. The script for evaluation of real dimensions of the lattice structures in its each strut
- Digitized surface data were imported to the GOM Inspect software in theSTL file.

- The whole imported geometry was selected for it's fitting on the CAD using "Best fit alignment".

- The script used the strut's midpoints (CAD) as the centres of spheres for geometry/area selection ("Geometry-Based Selection"). This geometry/area were used for fitting of cylinders in the next step. This ensured the same selection for all struts in the lattice structures.

- In each strut, the script first created the Gauss cylinder. Then the inscribed and circumscribed cylinders were created on the same selection using the direction of the Gauss cylinder axis for definition of their axes direction.

- The dimensions of measured cylinders were exported to software Minitab for the next statistical analysis.
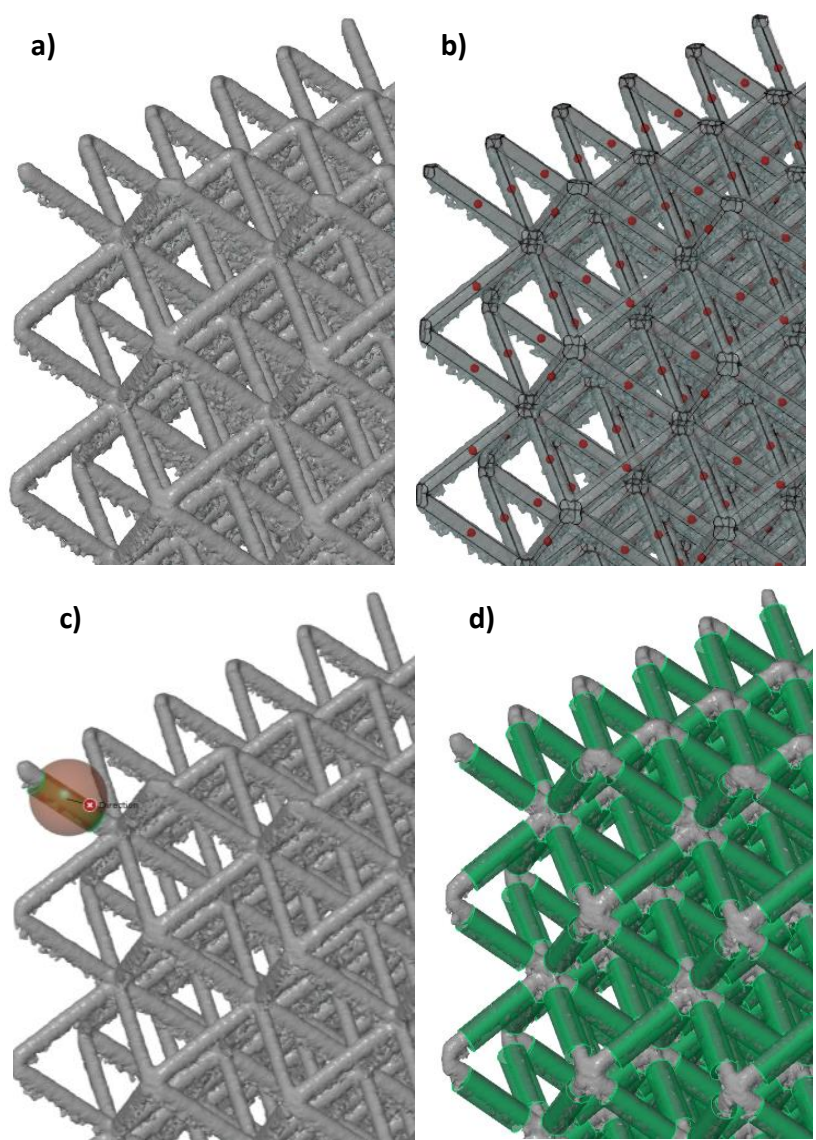

Figure 4. The process of evaluation of the real shape and dimensions by the semi-automatic script

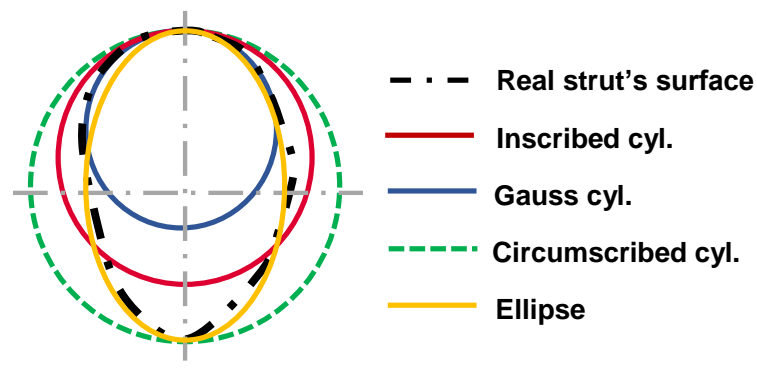

Figure 5. Schematic representation of evaluated ideal cylinders and ellipse

\section{RESULTS AND DISCUSSION}

\subsection{Shape and dimensional analysis}

The detailed statistical results of all analysed specimens are shown in Table 2. Generally, a trend is similar for all specimens; 
therefore, the results will be presented only on the LC-02 specimen with struts dimensions in the middle of thetested range.

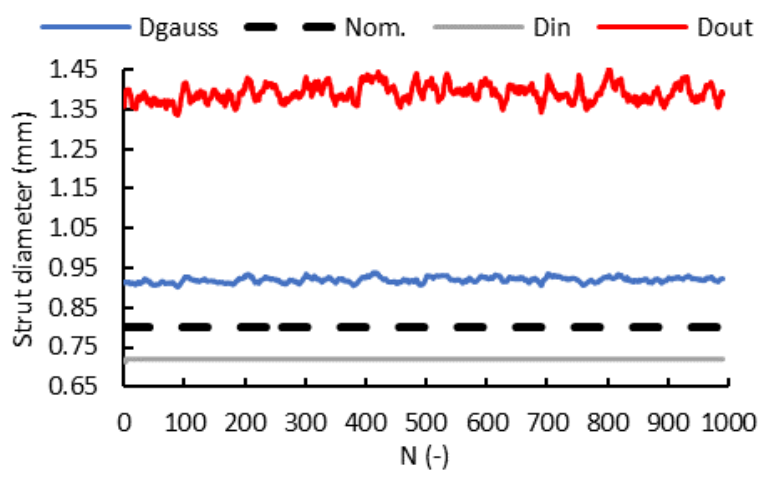

Figure 6. The results of three measured cylinders measured in each strut of the LC-02 lattice cube

Due to using outputs of $\mu \mathrm{CT}$ analysis together with semiautomatic shape analysis, the large number of values were obtained for each lattice cube (for LC-02; $N=1000$ ). Unfortunetaly, the semi-automatic script does not allow to fully control the fitting process of the cylinders and the results could be slightly deviated due to possible defects in the measured surface data. Therefore, the basic average filter from 10 values was applied. Then, the results of the inscribed, Gauss and circumscribed cylinders were visualized together with the nominal diameter in Figure 6. The chart showed large deviations between inscribed and circumscribed cylinders, specifically of $0.683 \mathrm{~mm}$ in case of median values.

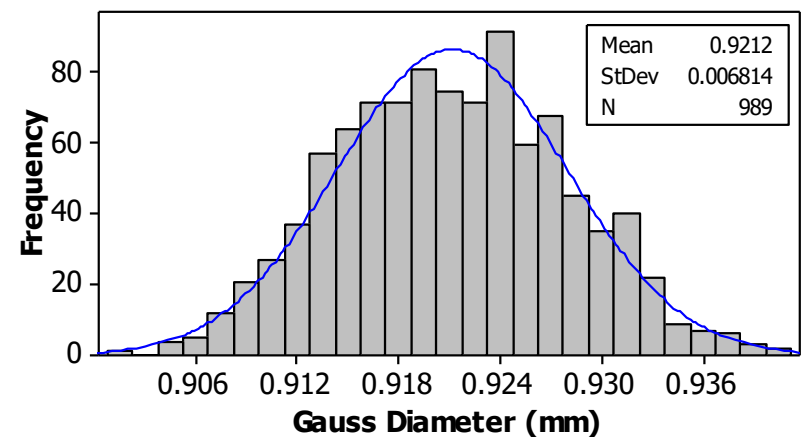

Figure 7. Frequency of strut diameters distribution of the LC-02 specimen

A frequency of the Gauss cylinders dimensions is presented in Figure 7. Here is shown that the values have the normal distribution with a range of $0.039 \mathrm{~mm}$ and a median value of $0.921 \mathrm{~mm}$. The Gauss dimensions were also put into chart Figure 9 which shows the dependence of the strut diameter on the produced $Z$ height. The $Z$ height is expressed by the variable $\boldsymbol{N}$, where $\boldsymbol{N} \leq 200$ represent the first layer of the lattice structure, $200<\boldsymbol{N} \leq 400$ represent the second layer and etc. (Figure 8). The results show that in the first layer of the lattice structure, the strut diameter was increased. From the second layer, the diameter is stabilized and its mean and average values are constant in the rest of $Z$ height. It is caused by heat transfer through the lattice structure. In the first layer $(\boldsymbol{N} \leq 200)$, the residual heat energy produced during the SLM process is transfered directly to the platform through the inclined struts (in angle $35.26^{\circ}$ ). In the second layer, the orientation of each strut is changed about $90^{\circ}$ (to the angle $\left.-35.26^{\circ}\right)$; therefore, the heat transfer is lower. Consequently, the heat energy is accumulated in the lattice structure and influence the surrounding powder which is melted. This increase the diameter of the lattice structure, especially the circumscribed diameter.

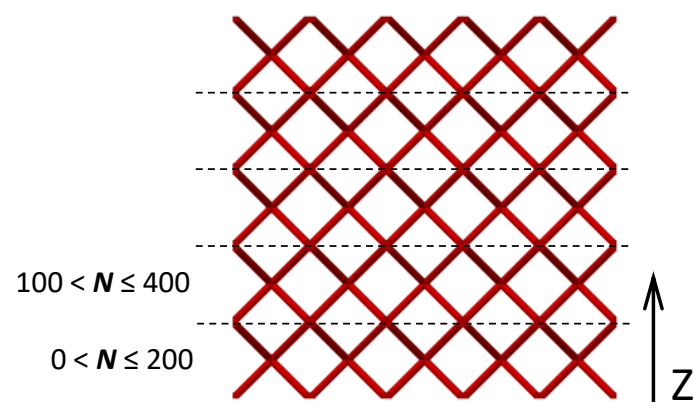

Figure 8. Schema of the $\boldsymbol{N}$ parameter placement in the lattice structure cube

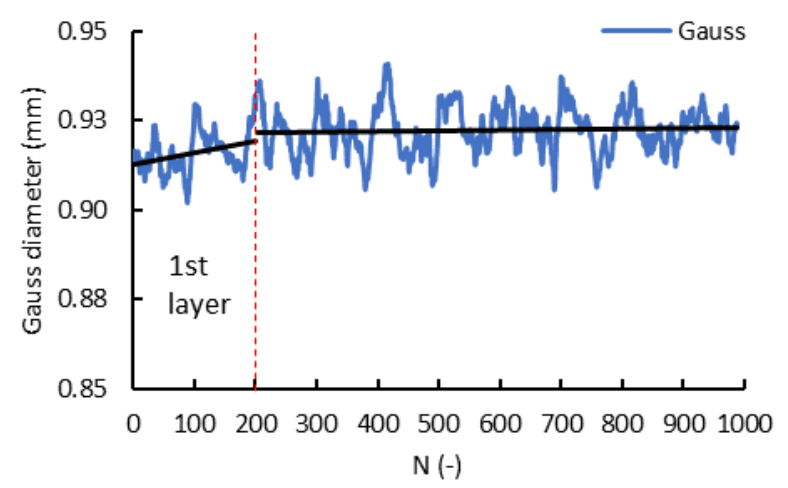

Figure 9. The change of the lattice structure diameter in the first layer of the lattice structure

\begin{tabular}{|c|c|c|c|c|}
\hline & $\begin{array}{l}\text { Parameters - } \\
\text { Specimens }\end{array}$ & LC-01 & LC-02 & LC-03 \\
\hline & $N(-)$ & 2733 & 989 & 501 \\
\hline & Dout (mm) & 0.771 & 0.941 & 1.022 \\
\hline & $\operatorname{Dmin}(\mathrm{mm})$ & 0.721 & 0.902 & 0.994 \\
\hline & Range (mm) & 0.051 & 0.039 & 0.028 \\
\hline & Median (mm) & 0.741 & 0.921 & 1.009 \\
\hline రृ & Dout (mm) & 0.589 & 0.721 & 0.833 \\
\hline 도. & Dmin $(\mathrm{mm})$ & 0.536 & 0.656 & 0.763 \\
\hline 气ू & Range (mm) & 0.052 & 0.065 & 0.070 \\
\hline & Median (mm) & 0.566 & 0.707 & 0.809 \\
\hline 亡் & Dout (mm) & 1.232 & 1.461 & 1.567 \\
\hline हᄐ & Dmin $(\mathrm{mm})$ & 0.979 & 1.336 & 1.366 \\
\hline 을 & Range (mm) & 0.254 & 0.125 & 0.201 \\
\hline & Median (mm) & 1.103 & 1.390 & 1.405 \\
\hline
\end{tabular}

Table 2. The global dimensional parameters of the analysed lattice specimens

\subsection{Shape Analysis Using Semi-automatic Script}

The results from the previous analysis showed significant deviations between inscribed and circumscribed cylinders as is shown in Figure 5 and also in Figure 6 . It is caused by lattice structure shape inaccuracy due to particular heat transfer to surrounding powder during SLM production. The heat transfer occurs mainly in the thermal gradient direction (negative $Z$ direction); therefore, the powder is sintered mainly on the down-skin surface of the lattice structure.

Because the shape inaccuracy occurred mainly in one direction, (thermal gradient) an elliptic shape better corresponds with a real cross-section of the strut. Therefore, the semi-automatic script was modified as follow: 
- Already created Gauss cylinders were used for the analysis of ellipticity.

- The perpendicular planes to the Gauss cylinder axis were created in its start, mid and end points.

- The planes were used to create the single cross-section to obtain the 2D geometry of the strut

- The ellipses were fitted into 2D geometry

- The final dimensions of each strut were obtained as the arithmetic average from three ellipses.

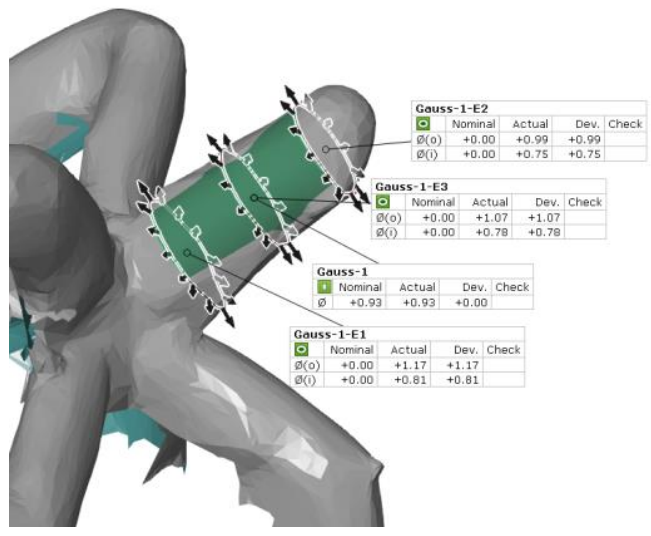

Figure 10. Evaluation of the elliptic cross-section of the lattice structures struts

\begin{tabular}{|c|c|c|c|}
\hline LC-02 & Minor axis & Major axis & Ellipticity \\
\hline Max $(\mathrm{mm})$ & 1.06 & 1.67 & - \\
\hline Min $(\mathrm{mm})$ & 0.69 & 0.85 & - \\
\hline Range $(\mathrm{mm})$ & 0.37 & 0.82 & - \\
\hline Median $(\mathrm{mm})$ & 0.79 & 1.10 & 0.717 \\
\hline
\end{tabular}

Table 3. The obtained results of ellipticity of the LC-02 specimen

The obtained data confirmed the strut deformation in build negative $Z$ drection. This expresed ratio between minor and major axes of the ellipse called ellipticity. Next, the crosssection areas of elliptical and circular struts were compared $A_{\text {Din }}=0.393 \mathrm{~mm}^{2}$ (Inscribed cylinder), $A_{\text {Dout }}=1.517 \mathrm{~mm}^{2}$ (the circumscribed cylinder), $A_{\text {Gauss }}=0.666 \mathrm{~mm}^{2}$ (Gauss cylinder) and $A_{e}=0.675 \mathrm{~mm}^{2}$ (ellipse cross-section). It showed that from different kinds of measured cylinders the Gauss cylinder best represent the real geometry of the lattice structure. This confirmes the study presented by [Suard 2014].

The shape modification occurred due to the SLM procedural inaccuracy strongly influence the mechanical properties of the lattice structures. Therefore, the results of the shape and dimensional analyses were used in FEA model presented in [Vrana 2018b] to quantify the deviations between the prediction and real experiment depending on the used cross-section geometry. The numerical results showed that the elliptic cross-section better represents the real mechanical behaviour of lattice structure when the $\boldsymbol{F}_{\max }$ deviation from the experimental test was only $2 \%$ (Figure 12). In the case of the circular cross-section, it was $12 \%$ and also the course of the line corresponded not so well with experiment (Figure 11).

\section{CONCLUSION}

- In the presented article, three lattice structure specimens with a constant relative density of $5 \%$ and a diameter between of $0.6-1.0 \mathrm{~mm}$ were designed and produced by SLM

- The specimens were digitized in the whole volume by $\mu \mathrm{CT}$

- The shape and dimension analyses were performed using the developed semi-automatic script in software GOM Inspect.
The script evaluated three types of cylinders and ellipses in each strut of the lattice structures

- The obtained data were analysed and the global dimension parameters for all specimens were determined.

- The results showed the different diameter of the lattice structures struts in the first layer. This is caused by better heat transfer to the platform than in the rest of the lattice structure.

- The results also showed a large deviation between circumscribed and inscribed diameter. It is caused by an accumulation of the residual heat energy of the SLM process which caused melting of the metal powder mainly around the down-skin surface of the lattice structure.

- The results of numerical simulation showed that the deviation from the experimental results is about $10 \%$ lower in the case of elliptical lattice structure compared to circular.

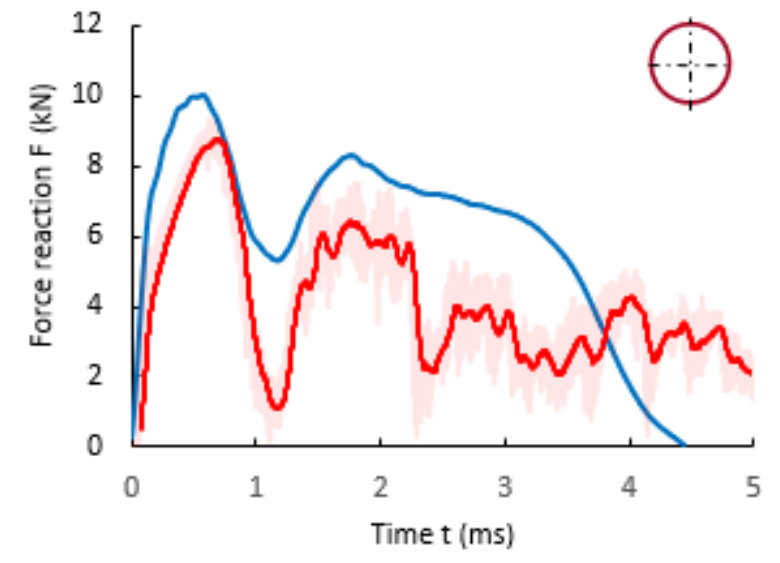

Figure 11. Comparison of the experimental results (blue curve) and the numerical simulation (red curve) with circular cross-section [Vrana 2018b]

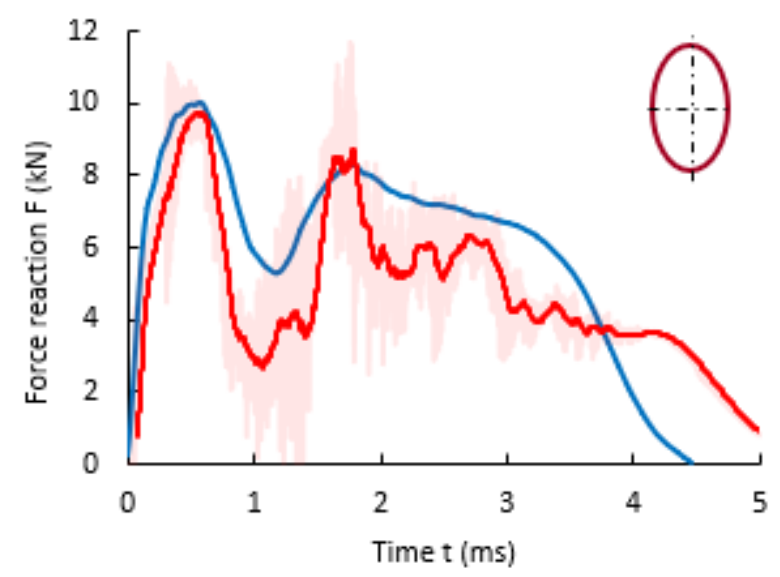

Figure 12. Comparison of the experimental results (blue curve) and the numerical simulation (red curve) with elliptical cross-section [Vrana 2018b]

\section{ACKNOWLEDGMENTS}

This paper is an output of research and scientific activities covered by:

- TACR project TH02010514, Development of 3D printing for selected materials and topology optimization of components for aerospace industry

- ESIF, EU Operational Programme Research, Development and Education within the research project [Architectured materials designed for additive manufacturing] grant number [CZ.02.1.01/0.0/0.0/16_025/0007304]. 


\section{REFERENCES}

[Koutny 2014] Koutny, D. and Vrana, R. Dimensional accuracy of single beams of AlSi10Mg alloy and $316 \mathrm{~L}$ stainless stee manufactured by SLM. In: I. Drstvenšek, ed. 5th International Conference on Additive Technologies iCAT2014, Ljubljana: Interesansa, pp 142-147, ISBN 978-961-281-579-0.

[Leary 2016] Leary, M. and Mazur, M. Selective laser melting (SLM) of AlSi12Mg lattice structures. 2016. Vol. 98. pp 344-357. ISSN 02641275.

[Qiu 2015] Qiu, Ch. and Yue, Sh. Influence of processing conditions on strut structure and compressive properties of cellular lattice structures fabricated by selective laser melting. 2015. Vol. 628. pp 188-197. ISSN 0921-5093.

[Schleifenbaum 2010] Schleifenbaum, H. and Meiners, K. Individualized production by means of high-power Selective Laser Melting. 2010. Vol. 2. No. 3. pp 161-169. ISSN 17555817.
[Suard 2014] Suard, M. and Lhuissier, R. Impact of EBM Fabrication Strategies on Geometry and Mechanical Properties of Titanium Cellular Structures. In: A. Demmer, ed. Proceedings of the Fraunhofer Direct Digital Manufacturing Conference 2014, Berlin: Fraunhofer Verlag, pp 1-6, ISBN 978-3-8396-91281.

[Vrana 2016] Vrana, R. and Koutny. D. Impact Resistance of Different Types of Lattice Structures manufactured by SLM. 2016. No. 6. pp 1579-1585. ISSN: 1803-1269.

[Vrana 2018a] Vrana, R. and Koutny, D. Selective Laser Melting Laser Strategy for Fabrication of Thin Struts Usable in Lattice Structures. 2018. Vol. 11. No. 9. pp 1-20. ISSN 1996-1944.

[Vrana 2018b] Vrana, R. and Cervinek, O. Dynamic Loading of Lattice Structure Made by Selective Laser Melting-Numerical Model with Substitution of Geometrical Imperfections. 2018. Vol. 11. No. 11. pp 1-21. ISSN 1996-1944.

\section{CONTACTS}

Ing. Radek Vrana Ph.D.

Brno University of Technology

Faculty of Mechanical Engineering

Technická 2896/2. Brno. 616 69. Czech Republic

+420 54114 4927. radek.vrana@vut.cz.www.3dlaboratory.cz 